\title{
Ideal structure of the Kauffman and related monoids
}

\author{
Kwok Wai Lau ${ }^{1}$ and D. G. FitzGerald ${ }^{2}$ \\ ${ }^{1}$ School of Biological Sciences, University of Sydney \\ $2_{\text {School of Mathematics and Physics, University of Tasmania (address for correspondence) }}$
}

July 18, 2007

\begin{abstract}
The generators of the Temperley-Lieb algebra generate a monoid with an appealing geometric representation. It has been much studied, notably by Louis Kauffman. Borisavljević, Došen and Petrić gave a complete proof of its abstract presentation by generators and relations, and suggested the name 'Kauffman monoid'. We bring the theory of semigroups to the study of a certain finite homomorphic image of the Kauffman monoid. We show the homomorphic image (the Jones monoid) to be a combinatorial and regular *-semigroup with linearly ordered ideals. The Kauffman monoid is explicitly described in terms of the Jones monoid and a purely combinatorial numerical function. We use this to describe the ideal structure of the Kauffman monoid and two other of its homomorphic images.

Key Words: Kauffman monoid, ideal structure

2000 Mathematics Subject Classification: 20M20
\end{abstract}

\section{Introduction}

In discussing the relationships between problems of several types, including combinatorial enumeration and lattice spin problems, Temperley and Lieb [7] introduced operators here denoted $h_{i}$ which turned out to satisfy

$$
\begin{aligned}
h_{i}^{2} & =c h_{i}=h_{i} c, \\
h_{i} h_{j} & =h_{j} h_{i} \quad \text { for }|i-j| \geq 2, \\
h_{i} h_{j} h_{i} & =h_{i} \quad \text { for }|i-j|=1,
\end{aligned}
$$

for a parameter $c \in \mathbb{C}$ and $i, j=1 \ldots n-1$. An algebra $T L_{n}$ generated (as an algebra) by the $h_{i}$ subject to the relations (1) is thus called a Temperley-Lieb algebra. The basis elements of a Temperley-Lieb algebra constitute a monoid $K_{n}$ generated by $c, h_{1}, \ldots h_{n-1}$ and subject to the relations (1), so that the Temperley-Lieb algebra is the semigroup algebra $\mathbb{C}\left[K_{n}\right]$ of $K_{n}$.

A representation of the monoid $K_{n}$ by isotopy classes in point-set topology was found and popularised, perhaps most notably by Jones, in section 4 of [4], and by Kauffman [5]. In fact it may be described as combinatorial, in that it is almost entirely unnecessary to worry about isotopy, most proofs and computations needing only elementary ideas of planar graphs. In this representation, 
described in the next section, $c$ is represented by a generator called the circle element, and the $h_{i}$ by elements to be called hooks or (more formally) diapsides.

It was widely held that this representation was in fact an isomorphism, but no complete proof of this was published until Borisavljević, Došen and Petric [1]. They suggested the name Kauffman monoid for $K_{n}$ to honour the contributions of L. H. Kauffman to the theory of $K_{n}$ and $\mathbb{C}\left[K_{n}\right]$. More recently still, Došen and Petrić [2] discuss countable versions of this and related monoids, giving several representations including by friezes, bracketings and relatively free monogenic categories of a certain kind. We follow, where possible, the notation and nomenclature of these two last-mentioned papers.

Despite this attention, there seems to have been little investigation of the semigroup properties of $K_{n}$ and its relatives. This is what we attempt here. Our approach uses the quotient semigroup $J_{n}=K_{n} /(c, 1)^{\sharp}$ obtained by setting $c=1$ in relations (1), which we name here the Jones monoid (with thanks to an anonymous referee for the suggestion. The corresponding algebra $\mathbb{C}\left[J_{n}\right]$ is a quotient algebra of $T L_{n}$, namely $T L_{n} /\langle c-1\rangle$. We also discuss the nilpotentgenerated monoid $N_{n}=K_{n} /(c, 0)^{\sharp}$ obtained by setting $c=0$ in relations (1). These simple cases may help shed a little more light on algebras of greater physical relevance. The key results in section 3 were obtained in the first author's 2004 MAppSc project at the University of Tasmania.

\section{Diagrams}

Most of the descriptive material in this section is drawn from the sources above, but we sketch the facts briefly in a form which is convenient for our approach. Let $n \in \mathbb{N}^{+}$, and set $\mathbf{n}=\{1, \ldots n\}$. A diagram $a$ is a (undirected, loop-free) graph with the following specifications. The vertex set consists of the $2 n$ points of the plane, $\{(i, 0)(i, 1): i=1 \ldots n\}$, and the edges are drawn without intersection within the rectangle

$$
[1, n] \times[0,1]=\{(x, y): 1 \leq x \leq n, 0 \leq y \leq 1\} \subset \mathbb{R}^{2}
$$

in such a way that each vertex has degree one (so the graph is a complete matching). See Fig. 1. for examples.

The induced subgraph on the vertices $\{(i, 1)\}$ is called the top of $a$ and denoted $T(a)$; the induced subgraph on $\{(i, 0)\}$ is the bottom of $a$, denoted $B(a)$. Each of $T(a), B(a)$ is a partial matching on $n$ vertices, and $(i, 1)$ may be referred to simply as the vertex $i$ in $T(a)$ (and so on) without risking confusion. Likewise, we may refer to an edge joining vertices $(i, 1)$ and $(j, 1)$ as the edge $\{i, j\}$ in $T(a)$, etc. The edges in $T(a)$ or $B(a)$ are called arches and the edges with one vertex in each of $T(a)$ and $B(a)$ are called transversals. A partial matching will be called admissible if it occurs as the top or bottom of a diagram.

Recall that two diagrams are declared isotopic if there is a continuous map fixing the vertices which carries one diagram to the other. The set of isotopy classes of diagrams (for fixed $n$ ) may be endowed with a multiplication in several ways, leading to the monoids treated here. The basic idea is to copy the left (or upper) factor $s$ into the rectangle $[1, n] \times[1,2]=\{(x, y): 1 \leq x \leq n, 1 \leq y \leq 2\}$ by a translation $(x, y) \mapsto(x, y+1)$, glue it to the diagram of the right (or lower) 

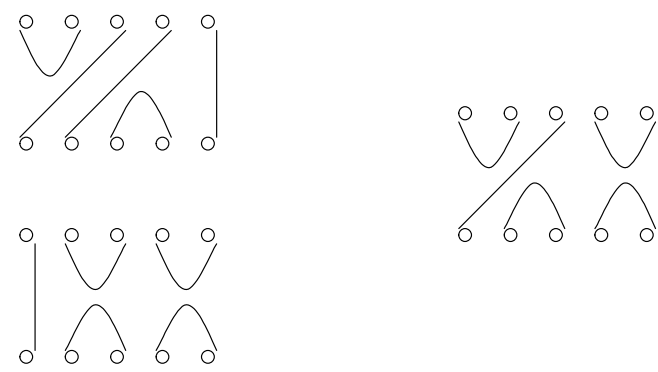

Figure 1: Two diagrams ( $s$ above and $t$ below), and their product st. The top graph of $s$ has just one edge, $\{1,2\}$. The bottom graph of $t$ has edges $\{2,3\}$ and $\{4,5\}$.

factor $t$, and then shrink vertically by the transformation $(x, y) \mapsto\left(x, \frac{y}{2}\right)$. Fig.1 has an example.

This operation is respected by isotopy, and is associative on isotopy classes. So we may simply work with diagrams which represent their respective isotopy classes. Note that circles arise in such compositions of diagrams, when the same pair of vertices are incident with edges in both $B(s)$ and $T(t)$. Variations on the basic theme, concerned with how such circles are treated, now lead to some of the monoids considered in [2]. In the multiplication for $K_{n}$, any part of a diagram which is (isotopic with) a circle is allowed to pass through any transversal or other circle. In the multiplication for $J_{n}$, all circles are erased. In the multiplication for $L_{n}$, a circle is not allowed to pass through either a transversal or another circle. In $N_{n}$, all diagrams containing a circle are identified with a zero element. In this paper we shall be chiefly concerned with $J_{n}$ and $K_{n}$.

The Jones monoid $J_{n}$. As noted in the introduction, [1] definitively established relations (1) as a presentation for $K_{n}$. A presentation for $J_{n}$ is obtained by adjoining $c=1$ to the relations (1) and is equivalent to the monoid presentation with generators $h_{i}$ for $i=1, \ldots n-1$ and relations

$$
\begin{aligned}
h_{i}^{2} & =h_{i}, \\
h_{i} h_{j} & =h_{j} h_{i} \quad \text { for }|i-j| \geq 2, \\
h_{i} h_{j} h_{i} & =h_{i} \quad \text { for }|i-j|=1 .
\end{aligned}
$$

The generator $h_{i}$ is represented by the diagram with arches $\{(i, 1),(i+1,1)\}$ and $\{(i, 0),(i+1,0)\}$ in $T\left(h_{i}\right)$ and $B\left(h_{i}\right)$ respectively, and transversals $\{(j, 1),(j, 0)\}$ for $j \neq i, i+1$ - see Fig. 2 .

The presentation above is apparently folklore, but a nice (Burnside-like) proof is outlined in [2]. In that proof we see that $J_{n}$ is finite (in fact, that the cardinality of $J_{n}$ is the $n$th Catalan number). The presentation also makes explicit that $J_{n} \cong K_{n} / \rho$, where $\rho$ is the congruence generated by the ordered pair $(c, 1)$. We use the same notation for the generators $h_{i}$ in both $J_{n}$ and $K_{n}$, and likewise write multiplication in both monoids as juxtaposition, relying on the context to distinguish them. We note here that with this convention, each 


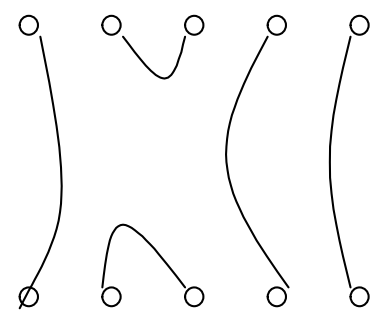

Figure 2: The generator $h_{2}$ in $H_{5}$.

element of $K_{n}$ may be written in the form $c^{k} w$ for some $k \in \mathbb{N}$ and $w \in J_{n}$. More formally,

Theorem 2.1 There is a bijection $\psi: \mathbb{N} \times J_{n} \rightarrow K_{n}$ defined by $\psi(k, w)=c^{k} w$ which becomes a homomorphism when multiplication in $\mathbb{N} \times J_{n}$ is defined by

$$
(k, u)(l, v)=(k+l+\gamma(u, v), u v)
$$

where $\gamma(u, v)$ is the number of new circles created in the product uv.

In section 4 below, we study the function $\gamma$, which together with $J_{n}$ provides all information about $K_{n}$ and therefore $T L_{n}$. First we must describe the structure of $J_{n}$, which is carried out below.

More about the top and bottom graphs of a diagram. We will often use the following lemma, sometimes without explicit reference.

Lemma 2.2 Let $s, t \in J_{n}$.

(i) $T(s)$ and $B(s)$ have equal numbers of edges, and $T(s) \subseteq T(s t)$.

(ii) For any admissible partial matchings $P$ and $Q$ on a vertex set $\mathbf{n}=\{1, \ldots n\}$ having equal numbers of edges, there exists a unique $s \in J_{n}$ such that $T(s)=P$ and $B(s)=Q$.

Proof. Part (i) is immediate from the definitions. Part (ii): clearly the unmatched vertices of $P$ and $Q$ are equally numerous; we may represent them as the point sets $\left\{\left(a_{i}, 1\right)\right\}$ and $\left\{\left(b_{i}, 0\right)\right\}$ with $a_{i}, b_{i} \in \mathbb{N}$ for $i=1 \ldots r$ and

$$
1 \leq a_{1}<a_{2} \ldots<a_{r} \leq n, \quad 1 \leq b_{1}<b_{2} \ldots<b_{r} \leq n .
$$

Any $s$ as specified in the statement must have edges joining $\left(a_{i}, 1\right)$ to $\left(b_{i}, 0\right)$ for $i=1 \ldots r$, and there is at most one such $s$, since planarity ensures the ordering is preserved. So it remains only to show, by construction, that this $s$ exists. Now the edges of $P$ and $Q$ may be drawn within the strips $\frac{2}{3} \leq y \leq 1$ and $0 \leq y \leq \frac{1}{3}$ respectively. Because $P$ and $Q$ are admissible it is possible to draw, without crossings, edges joining $\left(a_{i}, 1\right)$ to $\left(a_{i}, \frac{2}{3}\right)$ and $\left(b_{i}, 0\right)$ to $\left(b_{i}, \frac{1}{3}\right)$ for each $i$, and then to complete a diagram for $s$ by drawing edges joining $\left(a_{i}, \frac{2}{3}\right)$ to $\left(b_{i}, \frac{1}{3}\right)$ in the strip $\frac{1}{3} \leq y \leq \frac{2}{3}$.

Define, for $s \in J_{n}$, a diagram $s^{*} \in J_{n}$ obtained by 'turning $s$ upside-down'; more formally, by reflection of the diagram for $s$ in the line $y=\frac{1}{2}$. We note that $T\left(s^{*}\right)=B(s)$, etc. 
Lemma 2.3 For $s, t \in J_{n}$,

(i) $s^{* *}=s$;

(ii) $(s t)^{*}=t^{*} s^{*}$;

(iii) $s s^{*} s=s$;

(iv) $s^{2}=s=s^{*}$ if and only if $T(s)=B(s)$.

Proof. Parts (i) to (iii) are verified by checking equality of the respective edgesets. For part (iv) we use Lemma 2.2 to identify $s^{*}$ as the unique element having $T\left(s^{*}\right)=B(s)$ and $B\left(s^{*}\right)=T(s)$, and then note that $T(s)=B(s)$ also implies $s^{2}=s$.

In the language of semigroup theory, parts (i) to (iii) affirm that $J_{n}$ is a regular *-semigroup [6]. We now state some useful facts about regular $*_{-}$ semigroups. An element $p$ satisfying $p^{2}=p=p^{*}$ is called a projection, and projections (and more generally idempotents) may be partially ordered by

$$
p \leq q \text { iff } p q=p=q p .
$$

Proposition 2.4 In a regular ${ }^{-}$-semigroup $S$,

(i) $x x^{*}$ is a projection and every projection is of this form (trivially, since $\left.p=p p^{*}\right)$;

(ii) every idempotent in $S$ is the product of two projections (in fact, $e=$ $\left.\left(e e^{*}\right)\left(e^{*} e\right)\right)$;

(iii) for $x, y \in S, x \in S y$ if and only if $x^{*} x \leq y^{*} y$ (in fact, $x=x y^{*} y \Longleftrightarrow$ $\left.x^{*} x=x^{*} x y^{*} y=y^{*} y x^{*} x\right)$.

\section{Ideal structure of $J_{n}$}

The ideal structure of $J_{n}$ will be described with the aid of Green's relations. What we need of these tools of semigroup theory is outlined in a couple of paragraphs here; for more detail, the reader may consult e.g. Howie [3]. Elements $a, b$ of a semigroup are said to be $\mathcal{L}$-related if they generate the same principal left ideal, that is, if $\{a\} \cup S a=\{b\} \cup S b$. Dually, $a, b$ are $\mathcal{R}$-related if $\{a\} \cup a S=\{b\} \cup b S$. Generation of the same principal two-sided ideal defines the relation $\mathcal{J}$. In a monoid, $(a, b) \in \mathcal{L}$ if and only if $S a=S b$, and dually for $\mathcal{R}$, while $(a, b) \in \mathcal{J}$ if and only if $S a S=S b S$. Define $\mathcal{D}=\mathcal{L} \circ \mathcal{R}=\mathcal{R} \circ \mathcal{L}$; it is the finest equivalence containing both $\mathcal{L}$ and $\mathcal{R}$.

Each $\mathcal{D}$-class in $S$ is simultaneously a disjoint union of $\mathcal{R}$-classes and of $\mathcal{L}$-classes, and if these are represented in rows and columns respectively, the $\mathcal{D}$ class has a rectangular structure - the so-called 'egg-box' diagram. An $\mathcal{R}$-class and an $\mathcal{L}$-class have a non-empty intersection if and only if they are contained in the same $\mathcal{D}$-class, and such an intersection is an $\mathcal{H}$-class (that is, $\mathcal{H}=\mathcal{L} \cap \mathcal{R}$ ). The $\mathcal{H}$-classes containing idempotents are precisely the maximal subgroups of S.

On the way to determining these relations in $J_{n}$, we collect some more detailed combinatorial information about the effects of multiplication. For graphs $\Gamma, \Delta$ on the vertices $1, \ldots n$, we construct a (multi-)graph $\Gamma \oplus \Delta$ on the same vertex set, which has edge set equal to the union of the edge sets of $\Gamma$ and $\Delta$. If $\Gamma$ and $\Delta$ are partial matchings, then each connected component of $\Gamma \oplus \Delta$ is a path or a cycle. If we imagine the edges of $\Gamma \oplus \Delta$ to be coloured red or blue (or 
your favourite other colours) according as they came from $\Gamma$ or $\Delta$, we see that colours alternate, and so each cycle is of even length.

Now let $|\Gamma|$ denote the number of edges in $\Gamma, \gamma(\Gamma \oplus \Delta)$ be the number of cycles in $\Gamma \oplus \Delta, \omega_{\Gamma}(\Gamma \oplus \Delta)$ be the number of odd paths in $\Gamma \oplus \Delta$ which begin and end with $\Gamma$-edges, and $\omega(\Gamma \oplus \Delta)$ be the total number of odd paths in $\Gamma \oplus \Delta$ $\left(\operatorname{so} \omega(\Gamma \oplus \Delta)=\omega_{\Gamma}(\Gamma \oplus \Delta)+\omega_{\Delta}(\Gamma \oplus \Delta)\right)$.

A function $\gamma$ was introduced in Theorem 2.1. Use of the same notation is justified- $\gamma(s, t)$ really depends only on $\Gamma=B(s)$ and $\Delta=T(t)$ since $\gamma(s, t)=\gamma(B(s) \oplus T(t))$. Similarly, we may choose for the sake of brevity to write $\omega(s, t)$ for $\omega(B(s) \oplus T(t))$, and so on. We use these ideas to further examine what happens in a product $s t$.

Lemma 3.1 Let $s, t \in J_{n}$. Then

(i) the edges of $T(s t) \backslash T(s)$ are in 1:1 correspondence with the odd-length path components in $B(s) \oplus T(t)$ which begin and end with $T(t)$-edges;

(ii) the edges of $B(s t) \backslash B(t)$ are in 1:1 correspondence with the odd-length path components in $B(s) \oplus T(t)$ which begin and end with $B(s)$-edges;

(iii) $|T(s t)|=|T(s)|+\omega_{T(t)}(s, t)$ and $|B(s t)|=|B(t)|+\omega_{B(s)}(s, t)$;

(iv) $|T(s t)|=\frac{1}{2}(|B(s)|+|T(t)|+\omega(s, t))$.

Proof. (i) An edge from $i$ to $k$ within $T(s t) \backslash T(s)$ is composed of a transversal $\left\{i, j_{1}\right\}$ of $s$, a path component $\left(j_{1} \ldots j_{\ell}\right)$ of $B(s) \oplus T(t)$ which begins and ends with $T(t)$-edges, and a transversal $\left(k, j_{\ell}\right)$ of $s$. The stated correspondence is of $\{i, k\}$ with $\left(j_{1} \ldots j_{\ell}\right)$. Part (ii) is similar and (iii) follows directly. Then (iv) is obtained by adding the equations of part (iii).

Corollary 3.2 If $T(t) \subseteq B(s)$ then $T(s t)=T(s)$.

Proof. The hypothesis ensures $\omega_{T(t)}(s, t)=0$ and so $|T(s t)|=|T(s)|$. But also $T(s t) \supseteq T(s)$ and so these sets are equal.

Corollary 3.3 If $p, q \in J_{n}$ are projections, then $p \leq q$ if and only if $T(p) \supseteq$ $T(q)$.

Proof. If $p q=q p=p$ then $T(q) \subseteq T(p)$ by Lemma 2.2 (i). Conversely if $T(q) \subseteq T(p)=B(p)$ then by Corollary $3.2, T(p q)=T(p)$. Similarly $B(p q)=$ $B(p)$, and by Lemma 2.2 (ii), $p q=p$. Hence $p=p^{*}=q^{*} p^{*}=q p$ also, and $p \leq q$.

Corollary 3.4 $T\left(s s^{*}\right)=T(s)$ and $B\left(s^{*} s\right)=B(s)$.

Proof. Take $t=s^{*}$ in Lemma 3.1 and examine $B(s) \oplus T\left(s^{*}\right)=B(s) \oplus B(s)$; it contains only cycles and so $T\left(s s^{*}\right)=T(s)$, etc.

We may now state and prove

Theorem 3.5 For $s, t \in J_{n}$,

(i) $s \in J_{n} t$ if and only if $B(s) \supseteq B(t)$;

(ii) $s \in t J_{n}$ if and only if $T(s) \supseteq T(t)$;

(iii) $(s, t) \in \mathcal{L}$ if and only if $B(s)=B(t)$;

(iv) $(s, t) \in \mathcal{R}$ if and only if $T(s)=T(t)$;

(v) $(s, t) \in \mathcal{H}$ if and only if $s=t$;

(vi) $(s, t) \in \mathcal{D}$ if and only if $|T(s)|=|T(t)|$;

(vii) $\mathcal{D}=\mathcal{J}$

(viii) $s \in J_{n} t J_{n}$ if and only if $|T(s)| \geq|T(t)|$. 
Proof. (i) The "only if" statement is Lemma 2.2 (i). So for the converse, suppose that $B(s) \supseteq B(t)$. By Corollary $3.4 B\left(s^{*} s\right) \supseteq B\left(t^{*} t\right)$ and by 3.3 $s^{*} s \leq t^{*} t$. Then by 2.4 (iii) $s \in J_{n} t$. Now (ii) is dual to (i) and (iii), (iv) are deduced from (i) and (ii) respectively. Since $\mathcal{H}=\mathcal{L} \cap \mathcal{R},(\mathrm{v})$ is a consequence of (ii) and (iv) taken with Lemma 2.2.

(vi) Suppose $(s, t) \in \mathcal{D}$. Then there is $u \in J_{n}$ such that $(s, u) \in \mathcal{L}$ and $(u, t) \in \mathcal{R}$. By (iii) and (iv), $B(s)=B(u)$ and $T(u)=T(t)$; by Lemma 2.2 (i), $|B(u)|=|T(u)|$ so $|T(s)|=|B(s)|=|T(t)|$. Conversely suppose $s$ and $t$ are such that $|T(s)|=|T(t)|$; thus $|T(s)|=|B(t)|$. By Lemma 2.2 (ii) there exists $u \in J_{n}$ such that $T(s)=T(u)$ and $B(u)=B(t)$, which is to say that $(s, u) \in \mathcal{R}$ and $(u, t) \in \mathcal{L}$, whence $(s, t) \in \mathcal{D}$.

(vii) Since $J_{n}$ is finite, it is periodic and by Prop. 2.1.4 of [3], $\mathcal{D}=\mathcal{J}$.

(viii) If $s=u t v$ for $u, v \in J_{n}$, then by (ii) $T(s) \supseteq T(u t)$. Also by (i) $B(u t) \supseteq B(t)$ and it follows that $|T(s)| \geq|T(u t)|=|B(u t)| \geq|B(t)|=|T(t)|$. Conversely suppose that $|T(s)| \geq|T(t)|$. It is possible to remove edges from $T\left(s s^{*}\right)=T(s)$, maintaining an admissible partial matching, until a graph $\Gamma$ with $|T(t)|$ edges remains. Let $p$ be the projection with $T(p)=B(p)=\Gamma$; by construction, $T(p) \subseteq T\left(s s^{*}\right)$. Then by Corollary $3.3, s s^{*}=s s^{*} p=p s s^{*}$, whence $s=p s$. But $|T(p)|=|\Gamma|=|T(t)|$ and so $(p, t) \in \mathcal{D}$. In particular, $p=u t v$ for some $u, v \in J_{n}$ and so $s=p s=u t v s \in J_{n} t J_{n}$.

We find the following notation useful: let

$$
D_{k}=\left\{s \in J_{n}:|T(s)|=k\right\}, \text { for } 0 \leq k \leq\left\lfloor\frac{n}{2}\right\rfloor
$$

$D_{k}$ is a $\mathcal{J}$-class by parts (vi) and (vii) of Theorem 3.5. We may refer to $k=$ $|T(s)|$ as the depth of the $\mathcal{J}$-class $D_{k}$ or of its elements.

Corollary 3.6 $J_{n}$ is a finite, combinatorial, projection-generated regular * monoid whose ideals form a chain.

Proof. In this context, "combinatorial" means that all subgroups are trivial and this is affirmed by Theorem 3.5, part (v). By part (viii), the principal ideals of $J_{n}$ are linearly ordered, and (since every ideal is a union of the principal ideals it contains) the same is true for the set of all ideals. We have already remarked on the remaining properties.

Remark 3.7 The ideal of $J_{n}$ generated by $D_{d}$ consists of all elements of depth $d$ or more and $D_{d+1}$ is a maximum proper ideal of $D_{d}$. The Rees quotient semigroup $D_{d} / D_{d+1}$ (for $0 \leq d<\left\lfloor\frac{n}{2}\right\rfloor$ ) is called the principal factor of $J_{n}$ associated with the $\mathcal{J}$-class $D_{d}$ and is completely 0 -simple (see sections 3.1 and 3.2 of [3] for further details). Therefore it has a Rees matrix representation $D_{d} / D_{d+1} \cong \mathcal{M}^{0}(\{1\} ; I, \Lambda ; P)$, where

$$
I=\Lambda=\{\text { admissible partial matchings with } d \text { edges }\}
$$

and for $i \in I$ and $\lambda \in \Lambda$,

$$
P_{\lambda i}=\left\{\begin{array}{cc}
1 & \text { if } \quad \omega(i \oplus \lambda)=0 \\
0 & \text { otherwise. }
\end{array}\right.
$$

When $d=\left\lfloor\frac{n}{2}\right\rfloor, D_{d} \cong \mathcal{M}(\{1\} ; I, \Lambda ; P)$ where $P_{\lambda i}=1$ for all $\lambda, i$. 
Lemma 2.3 determined the projections in $J_{n}$ and showed that each element is regular. We conclude this section by using Lemma 3.1 to determine all idempotents and pairs of mutually inverse elements.

Lemma 3.8 In $J_{n}$,

(i) $s^{2}=s$ if and only if $\omega(s, s)=0$;

(ii) $s t s=s$ and $t s t=t$ if and only if $\omega(s, t)=\omega(t, s)=0$.

Proof. (i) The following are equivalent: $s^{2}=s ; B\left(s^{2}\right)=B(s)$ and $T\left(s^{2}\right)=$ $T(s) ; \omega_{T(s)}(s, s)=0=\omega_{B(s)}(s, s)=0 ; \omega(s, s)=0$.

(ii) The following are equivalent: sts $=s ; B(s t s)=B(s)$ and $T(s t s)=$ $T(s) ; \omega(s t, s)=0$ and $\omega(s, t s)=0 ; \omega(s, t)=\omega(t, s)=0$.

\section{Detailed study of the functions $\gamma$ and $\omega$ in $J_{n}$}

Further knowledge about the functions $\gamma, \omega$ helps in establishing other characteristics of $J_{n}$ and the ideal structure of $K_{n}$.

Lemma 4.1 Given $u, v \in J_{n}$, there exist $u^{\prime}, v^{\prime} \in J_{n}$ such that $u v=u^{\prime} v=u v^{\prime}$ and $\gamma\left(u^{\prime}, v\right)=\gamma\left(u, v^{\prime}\right)=0$. If $n$ is even and $u v \notin D_{\frac{n}{2}}$, or if $n$ is odd, then we may choose $u^{\prime} \mathcal{R} u$ and $v^{\prime} \mathcal{L} v$.

Proof. If $\gamma(u, v)=0$ there is nothing to prove. Otherwise take a cycle in $B(u) \oplus T(v)$ and let its set of vertices be $I$. If $I=\mathbf{n}$ we have $\gamma(u, v)=1$ and we also know that $n$ is even and that there are no transversals in $u v$, so that $v \in D_{\frac{n}{2}}$. We complete this case later on. If $I \neq \mathbf{n}$ there are vertices one unit apart, say $i \in I$ and $i+1 \notin I$. (The case $i \in I$ and $i-1 \notin I$ is similar.)

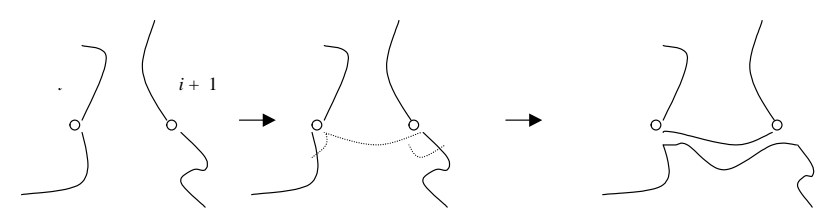

Figure 3: Modifying a diagram to decrease the number of cycles.

We prove the statements about $v^{\prime}$; duality (or a similar argument) proves the statements about $u^{\prime}$. Let the diagram be modified, preserving planarity, as follows. (See Fig.3.)

1. Draw an edge between $i$ and $i+1$ (possible since they are adjacent);

2. "perturb" this edge away from the vertices $i, i+1$, thus making a new edge from the mate of $i$ to the mate of $i+1$ (possible without crossings since there is a neighbourhood of the edge $(i, i+1)$ which does not intersect other edges); 3 . restore the edge $(i, i+1)$.

At the end of steps $1-3, u$ is unchanged but we have replaced $T(v)$ by an admissible partial matching $\Gamma_{1}$ with the same number of edges as $T(v)$. This defines a $v_{1} \in J_{n}$ such that

$$
T\left(v_{1}\right)=\Gamma_{1} \text { and } B\left(v_{1}\right)=B(v),
$$


from which we have $v_{1} \mathcal{L} v$. Moreover, the construction inserts all vertices in $I$ into the path or cycle including $i+1$, and so the set of transversals is unchanged. Thus both $u v=u v_{1}$, and $\gamma\left(u, v_{1}\right)=\gamma(u, v)-1$. Now repetition of this construction while there are vertices not in a cycle ends with a $v^{\prime} \in J_{n}$ such that $u v=u v_{1}=\cdots=u v^{\prime}$ and $v^{\prime} \mathcal{L} v$, and either (i) $\gamma\left(u, v^{\prime}\right)=0$ in which case we have finished, or (ii) $\gamma\left(u, v^{\prime}\right)=1$ and $B(u) \oplus T\left(v^{\prime}\right)$ is a cycle on $\mathbf{n}$, which is the same as the case we deferred above. So now we continue with case (ii).
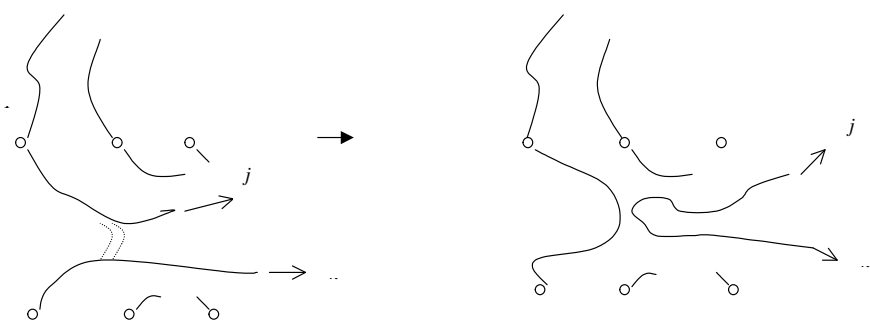

Figure 4: Removing the last cycle.

Let the vertex $1 \in T\left(v^{\prime}\right)$ be adjacent to $j$ in $T\left(v^{\prime}\right)$ and the vertex $1 \in B\left(v^{\prime}\right)$ be adjacent to $k$ in $B\left(v^{\prime}\right)$. (See Fig. 4.) Delete these edges, leaving admissible partial matchings on $T\left(v^{\prime}\right)$ and $B\left(v^{\prime}\right)$, since the original arches including the 1s were 'exterior' arches. $T\left(v^{\prime}\right)$ and $B\left(v^{\prime}\right)$ have equal numbers of edges and so define a diagram $v^{\prime \prime} \in J_{n}$, which includes transversals $\{1,1\}$ and $\{j, k\}$. (Thus $v^{\prime \prime}$ has depth $\frac{n}{2}-1$.) The graph $B\left(u v^{\prime \prime}\right)$ contains an arch $\{1, k\}$ arising from the path $(1,1, \ldots j, k)$, which now includes all vertices of the initial cycle. Therefore $B\left(u v^{\prime \prime}\right)=B\left(v^{\prime}\right)=B\left(u v^{\prime}\right)$ and $T\left(u v^{\prime \prime}\right)=T(u)=T\left(u v^{\prime}\right)$, so that $u v^{\prime \prime}=u v^{\prime}=u v$. Moreover $\gamma\left(u, v^{\prime \prime}\right)=\left(u, v^{\prime}\right)-1=0$.

The method of proof also gives a result dealing with the range of the function $\gamma$.

Corollary 4.2 For given $u \in J_{n}$ with $|T(u)|=m$, and each $l$ with $0 \leq l \leq m$, there is $u_{l} \in J_{n}$ such that $\gamma\left(u, u_{l}\right)=l$. If $m<\frac{n}{2}$ we may choose $u_{l} \in D_{m}$, and if $m=\frac{n}{2}$ we may choose $u_{l} \in D_{m-1}$. Since $\gamma(u, v) \leq|T(u)|=m$ and $1 \leq \gamma(u, v)$ for $u, v \in D_{\frac{n}{2}}$, these bounds are best possible.

Proof. In the Lemma, take $v$ to be $u^{*}$, and note that then $\gamma\left(u, u^{*}\right)=|B(u)|=$ $|T(u)|=m$. Then application of the lemma yields a sequence $v_{0}=u^{*}, v_{1}, \ldots v_{m}$ such that $\gamma\left(u, v_{m-l}\right)=l$ and the $v_{l}$ have the stated depths.

Next we investigate the range of the function $\omega(s, t)$ in terms of the depths of $s$ and $t$.

Lemma 4.3 $D_{k} D_{l} \cap D_{m} \neq \emptyset$ if and only if $m$ is such that $\max \{k, l\} \leq m \leq$ $\min \left\{k+l,\left\lfloor\frac{n}{2}\right\rfloor\right\}$. That is, the products of elements are distributed over exactly the $\mathcal{J}$-classes which their depths allow.

Proof. "Only if": clearly if $w=s t$ with $s \in D_{k}$ and $t \in D_{l}$, and $w$ has depth $m$, then $k, l \leq m \leq\left\lfloor\frac{n}{2}\right\rfloor$ by Theorem 3.5 part (viii). Also by 3.1 (iv),

$$
m \leq \frac{1}{2}(k+l+\omega(s, t)) \leq \frac{1}{2}(k+l+k+l)=k+l .
$$


"If": suppose $k, l \leq m \leq k+l,\left\lfloor\frac{n}{2}\right\rfloor$. We must find $s, t$ with the properties

$$
|B(s)|=k, \quad|T(t)|=l, \quad \omega(s, t)=2 m-k-l,
$$

the last requirement ensuring that $|T(s t)|=m$ by 3.1 (iv). Inspection shows that the choices

$$
\begin{aligned}
& s=h_{1} h_{3} \ldots h_{2 k-1}, \\
& t=h_{2 m-2 l+1} \ldots h_{2 m-1}
\end{aligned}
$$

have the desired properties.

For such a result as this lemma, we must be free to choose both factors: for example, in $J_{4}, D_{1} h_{2} \cap D_{2}=\emptyset$ even though $k=l=1$ and $m=2$ satisfy the inequalities.

Remark 4.4 These results could be framed alternatively in terms of the setvalued functions which give the ranges of the functions $\gamma$ and $\omega$ : let

$$
\begin{aligned}
& C(k, l)=\{\gamma(s, t):|T(s)|=k,|T(t)|=l\}, \\
& O(k, l)=\{\omega(s, t):|T(s)|=k,|T(t)|=l\} .
\end{aligned}
$$

Then $C(m, m)=\{0, \ldots m\}$ for $m \neq \frac{n}{2}, C\left(\frac{n}{2}, \frac{n}{2}\right)=\left\{1, \ldots \frac{n}{2}\right\}$ and $O(k, l)=$ $\{|k-l|, \ldots \min \{n-k-l, k+l\}\}$.

\section{Ideal structure of $K_{n}$ and two homomorphic images}

In this final section we bring information about the product-like structure of $K_{n}$ (Theorem 2.1) together with information about the local structure of $J_{n}$ (Theorem 3.5) to describe the ideal structure of $K_{n}$ and the two homomorphic images mentioned in the Introduction. We use Theorem 2.1 in the following equivalent but more convenient form: in $K_{n}$,

$$
\left(c^{m} s\right)\left(c^{n} t\right)=c^{(m+n+\gamma(s, t))} s t .
$$

Theorem 5.1 Let $c^{k} s, c^{l} t \in K_{n}$ with $k, l \in \mathbb{N}, s, t \in J_{n}$. Then

(i) $c^{k} s \in K_{n}\left(c^{l} t\right)$ if and only if $s \in J_{n} t$ and $k \geq l$;

(ii) $c^{k} s \in\left(c^{l} t\right) K_{n}$ if and only if $s \in t J_{n}$ and $k \geq l$;

(iii) $c^{k} s \in K_{n}\left(c^{l} t\right) K_{n}$ if and only if $s \in J_{n} t J_{n}$ and $k \geq l$.

Proof. (i) "Only if": suppose $c^{k} s=\left(c^{j} u\right)\left(c^{l} t\right)=c^{j+l+\gamma(u, t)} u t$. Then $k=$ $j+l+\gamma(u, t) \geq l$ and $s \in J_{n} t$.

"If": suppose conversely that $k \geq l$ and $s=u t$ for some $u \in J_{n}$. By Lemma 4.1 , there exists $u^{\prime} \in J_{n}$ such that $s=u^{\prime} t$ and $\gamma\left(u^{\prime}, t\right)=0$. Then

$$
\left(c^{k-l} u^{\prime}\right)\left(c^{l} t\right)=c^{k} u^{\prime} t=c^{k} s .
$$

(ii) This is the lateral dual of (i).

(iii) "Only if": suppose

$$
c^{k} s=\left(c^{j} u\right)\left(c^{l} t\right)\left(c^{m} v\right)=c^{j+l+m+\gamma(u, t)+\gamma(u t, v)} u t v .
$$


Then $k \geq l$ and $s \in J_{n} t J_{n}$.

"If": suppose conversely that $k \geq l$ and $s=u t v$ for some $u \in J_{n}$. By Lemma 4.1, there exist $u^{\prime}, v^{\prime} \in J_{n}$ such that $s=u^{\prime} t v^{\prime}$ and $\gamma\left(u^{\prime}, t\right)=\gamma\left(u^{\prime} t, v^{\prime}\right)=0$. Then

$$
\left(c^{k-l} u^{\prime}\right)\left(c^{l} t\right)\left(c^{0} v^{\prime}\right)=c^{k+\gamma\left(u^{\prime}, t\right)+\gamma\left(u^{\prime} t, v^{\prime}\right)} u^{\prime} t v^{\prime}=c^{k} s .
$$

Corollary 5.2 The lattice of principal ideals of $K_{n}$ is the product of a chain of length $\left\lfloor\frac{n}{2}\right\rfloor$ with a chain isomorphic to $\mathbb{N}$. All ideals of $K_{n}$ are finitely generated.

Proof. The ordering of the principal ideals in theorem 5.1 is the product ordering. Every ideal is generated by an antichain (set of incomparable elements), and every antichain in a product of chains with one factor finite has a finite number of elements (by the pigeonhole principle).

We proceed to treat $K_{n} /\left(c^{r}, 1\right)^{\sharp}$ similarly. Here the multiplication may be expressed as

$$
\left(c^{m} s\right)\left(c^{n} t\right)=c^{(m+n+\gamma(s, t)) \bmod r} s t .
$$

Theorem 5.3 Let $c^{k} s, c^{l} t \in K_{n} /\left(c^{r}, 1\right)^{\sharp}$, with $k, l \in \mathbb{N}, s, t \in J_{n}$. Then

(i) $c^{k} s \in K_{n}\left(c^{l} t\right)$ if and only if $s \in J_{n} t$;

(ii) $c^{k} s \in\left(c^{l} t\right) K_{n}$ if and only if $s \in t J_{n}$;

(iii) $c^{k} s \in K_{n}\left(c^{l} t\right) K_{n}$ if and only if $s \in J_{n} t J_{n}$.

Proof. The proof is the same as that of Theorem 5.1 except that $c^{r}=c^{0}$, and congruences $\bmod r$ such as $k \equiv j+l+\gamma(u, t)$ are always solvable for $j$, given the other variables.

Corollary 5.4 The lattice of ideals of $K_{n} /\left(c^{r}, 1\right)^{\sharp}$ is a chain of length $\left\lfloor\frac{n}{2}\right\rfloor$. Its egg-box diagram is that of $J_{n}$, except that each $\mathcal{H}$-class has $r$ elements and each maximal subgroup is cyclic of order $r$. The principal factor $D_{d}^{0}$ associated with the $\mathcal{J}$-class $D_{d}$ has the Rees matrix form $\mathcal{M}^{0}\left(\mathbb{Z}_{r} ; I, \Lambda ; P\right),\left(\right.$ or $\mathcal{M}\left(\mathbb{Z}_{r} ; I, \Lambda ; P\right)$ when $d=\left\lfloor\frac{n}{2}\right\rfloor$ ) with $I, \Lambda$ and $P$ as in Remark 4.3.

We now turn to the nilpotent-generated monoid $N_{n}$; in section 1 , this was introduced as the quotient $K_{n} /(c, 0)^{\sharp}$ obtained by setting $c=0$ in relations (1), but it is better dealt with as a modification of $J_{n}$, easily seen to be equivalent and described next. Take the base set of $J_{n}$ (the set of diagrams as defined in section 2), and adjoin an element 0 . Define a multiplication $\circ$ on $N_{n}=J \cup\{0\}$ by setting, for $s, t \in J_{n}, 0 \circ 0=s \circ 0=0 \circ s=0$ and

$$
s \circ t=\left\{\begin{array}{ccc}
0 & \text { if } & \gamma(s, t)>0, \\
s t & \text { if } & \gamma(s, t)=0,
\end{array}\right.
$$

juxtaposition meaning multiplication in $J_{n}$ as before. (Note that the abstract property of $\gamma$ which ensures associativity of multiplication on $\mathbb{N} \times J_{n}$ as described in Theorem 2.1 also guarantees that $\circ$ is associative on $N_{n}$.)

Theorem 5.5 Let $s, t \in N_{n} \backslash\{0\}$. Then

(i) $s \in N_{n} \circ t$ if and only if $s \in J_{n} t$;

(ii) $s \in t \circ N_{n}$ if and only if $s \in t J_{n}$;

(iii) $s \in t \circ N_{n} \circ t$ if and only if $s \in t J_{n} t$. 
Proof. If $s \in N_{n} \circ t$ then $0 \neq s=u \circ t=u t$ for some $u \in J_{n}$ whence $s \in J_{n} t$. Conversely, if $s \in J_{n} t$ then by Lemma 4.1, $s=u t$ for some $u \in J_{n}$ such that $\gamma(u, t)=0$. Then $s=u \circ t \in N_{n} \circ t$. Similarly for the rest.

Corollary 5.6 The lattice of ideals of $N_{n}$ is a chain of length $\left\lfloor\frac{n}{2}\right\rfloor+1$. Its eggbox diagram is that of $J_{n}$ with a zero adjoined. The principal factor associated with $D_{d}\left(d \leq\left\lfloor\frac{n}{2}\right\rfloor\right)$ has the form $\mathcal{M}^{0}(\{1\} ; I, \Lambda ; P)$, with $I$ and $\Lambda$ as before and

$$
P_{\lambda i}=\left\{\begin{array}{ccc}
1 & \text { if } & \gamma(i \oplus \lambda)=0 \\
0 & \text { otherwise. }
\end{array}\right.
$$

\section{References}

[1] Borisavljević, M.; Došen, K.; Petrić, Z. Kauffman monoids. J. Knot Theory Ramifications 2002, 11, 127-143.

[2] Došen, K.; Petrić, Z. Self-adjunctions and matrices. J. Pure Appl. Algebra 2003, 184, 7-39.

[3] Howie, J. M. Fundamentals of semigroup theory; Oxford University Press: Oxford, 1995.

[4] Jones., V. F. R. Index for subfactors. Invent. Math. 1983, 72, 1-25.

[5] Kauffman, L. H. An invariant of regular isotopy. Trans. Amer. Math. Soc. 1990, 318, 417-471.

[6] Nordahl, T. E.; Scheiblich, H. E. Regular *-semigroups. Semigroup Forum 1978, 16, 369-377.

[7] Temperley, H. N. V.; Lieb, E. H. Relations between the 'percolation' and 'colouring' problem and other graph-theoretic problems associated with regular planar lattices: some exact results for the 'percolation' problem. Proc. Roy. Soc. Lond. A 1971, 322, 251-280. 